\title{
Estudo da estabilidade dimensional da madeira de Cedro-marinheiro, Guarea trichilioides L.
}

\section{Study of dimensional stability on wood of Cedro-marinheiro, Guarea trichilioides $\mathbf{L}$.}

\author{
${ }^{1}$ Alan Felix Falavinha, ${ }^{2}$ Norman Barros Logsdon, ${ }^{3}$ Zenesio Finger, ${ }^{4}$ José Manoel Henriques de Jesus \\ ${ }^{1}$ Faculdade de Engenharia Florestal-FENF da Universidade Federal de Mato Grosso - UFMT \\ ${ }^{2}$ In memória*. Foi professor doutor UFMT, Programa de Mestrado em Edificações e Ambiental PPGEEA da \\ Faculdade de Arquitetura, Engenharia e Tecnologia-FAET e no Curso de Engenharia Florestal da FENF/UFMT \\ ${ }^{3}$ Professor Doutor da FENF, participa do Programa de Pós-graduação em Ciências Florestais e \\ Ambientais/UFMT \\ ${ }^{4}$ Professor Doutor, PPGEEA e no curso de Engenharia Civil e Sanitária da FAET/UFMT
}

Recebido em: Dezembro de 20165 Aceito em: março de 2016 Publicado em: Junho de 2016

\begin{abstract}
Resumo: A exploração de florestas nativas, em Mato Grosso, só é permitida por meio do manejo florestal, apenas as árvores maduras podem ser retiradas. Com a escassez de madeiras tradicionais outras espécies, de características desconhecidas, começaram a ser introduzidas no comércio. O objetivo deste trabalho foi reunir dados sobre as principais características físicas e dendrológicas, bem como estudar mais detalhadamente a estabilidade dimensional, traçando os diagramas de retração e inchamento, da madeira de Cedro-marinheiro, Guarea trichilioides L. Para descrição dendrológica e identificação da espécie foram utilizados os métodos tradicionais utilizados pela Dendrologia e Taxonomia. Para caracterização física utilizou-se a metodologia proposta por Logsdon (2002) para revisão da NBR 7190, da ABNT (1997). A partir de três árvores desta espécie foram coletados, no município de Cotriguaçu - MT, 12 corpos-de-prova, sendo quatro por árvore. Por meio de ensaios de estabilidade dimensional obtiveram-se: densidade aparente, ao teor de umidade de $12 \%$, de $\rho_{\text {ap, } 12 \%}=0,7506 \mathrm{~g} / \mathrm{cm}^{3}$; densidade básica de $\rho_{\text {bas }}=0,6124 \mathrm{~g} / \mathrm{cm}^{3}$; coeficientes de anisotropia dimensional, no inchamento, de $A_{i}=2,0945$, e na retração, de $A_{r}=1,9965$. Estes resultados sugerem mediana resistência mecânica, posicionando essa madeira na classe de resistência D30 ou D40 das dicotiledôneas, e que pode ser utilizada em estruturas de madeira de médio porte. Os coeficientes de anisotropia, por sua vez, sugerem madeira de qualidade normal, que apresenta alguns defeitos oriundos da secagem, mas pode ser usada na fabricação de móveis que aceitem pequenos empenamentos (mesas, cadeiras estantes etc.).
\end{abstract}

Palavras-chave: Retração. Inchamento. Densidade.

Abstract: The exploitation of native forests, in Mato Grosso, is only permitted by means of forest management; only the mature trees can be removed. With the scarcity of traditional timbers other species, whose characteristics are unknown, began to be introduced in the trade. The objective of this study was to gather data on the main physical and dendrological characteristics, as well as study in more detail the dimensional stability, drawing swelling and shrinkage diagrams to Cedro-marinheiro, Guarea trichilioides L. For dendrological description and identification of the species were used traditional methods used by Taxonomy and Dendrology. For physical characterization was used the methodology proposed by Logsdon (2002) for revision of NBR 7190, from ABNT (1997). From three trees of this species were collected, in the municipality of Cotriguaçu - MT, 12 specimens, four per tree. By means of dimensional stability tests were obtained: specific gravity, at $12 \%$ moisture content, $\rho_{\mathrm{ap}, 12 \%}=0.7506 \mathrm{~g} / \mathrm{cm}^{3} ;$ basic density, $\rho_{\mathrm{bas}}=0.6124 \mathrm{~g} / \mathrm{cm}^{3} ;$ dimensional anisotropy coefficients, on swelling, $A_{i}=2.0945$, and on shrinkage, $A_{r}=1.9965$. These results suggest medium mechanical strength, positioning this wood in resistance class D30 or D40, and can be utilized on medium timber structures. The anisotropy coefficients, suggests normal quality wood, which presents some defects during drying process, but can be used in furniture manufacture that accept small distortions (tables, chairs, shelves etc.).

Keywords: Shrinkage. Swelling. Specific gravity.

\section{INTRODUÇÃO}

Atualmente a exploração de florestas nativas, em Mato Grosso, só é permitida se existir manejo florestal tido como sustentável, ou seja, a floresta deve ser mantida e apenas as árvores de diâmetro, à altura do peito, superior a $40 \mathrm{~cm}$ podem ser retiradas. Isto tornou mais difícil a obtenção das madeiras tradicionalmente

\footnotetext{
* Professor Norman faleceu poucos dias antes da publicação do artigo
} 
empregadas e outras espécies, antes abandonadas na mata, começaram a ser introduzidas no comércio.

A maioria destas espécies tem suas características desconhecidas ou pouco conhecidas, como é o caso do Cedromarinheiro, Guarea trichilioides L., tornando relevante um trabalho de caracterização da madeira desta espécie.

Estudos relativamente recentes têm mostrado que os resultados obtidos no passado, até 1997, sobre a variação dimensional da madeira durante a secagem estão incorretos, pois partiam da hipótese errada de serem semelhantes os fenômenos de inchamento e retração da madeira (apenas mudaria o sentido de fluxo da água). Os dois fenômenos são distintos e, portanto, torna-se necessário obter os resultados sobre estabilidade dimensional de praticamente todas as espécies produtoras de madeira do Brasil.

O objetivo deste trabalho foi obter as principais características físicas e dendrológicas, incluindo os diagramas de inchamentos e de retrações, da madeira de Cedro-marinheiro, Guarea trichilioides L. A variação da densidade aparente com o teor de umidade também foi avaliada, tanto no umedecimento quanto na secagem.

\section{REVISÃO DE LITERATURA}

As principais características físicas da madeira podem ser obtidas a partir de ensaios de estabilidade dimensional. As dimensões da madeira se alteram com a variação da umidade, no intervalo de $0 \%$ até o limite de saturação das fibras. Neste intervalo, conhecido como intervalo higroscópico, ao aumentar o teor de umidade as dimensões da madeira aumentam (inchamento) e ao diminuir o teor de umidade as dimensões diminuem (retração). Segundo Kollmann e Côté Jr. (1984), o comportamento da madeira ao inchamento volumétrico é o apresentado na Figura 1 e nas Expressões (1) e (2). O mesmo comportamento é verificado para as deformações específicas no umedecimento (inchamentos lineares).

Figura 1 - Modelo de comportamento para inchamento volumétrico

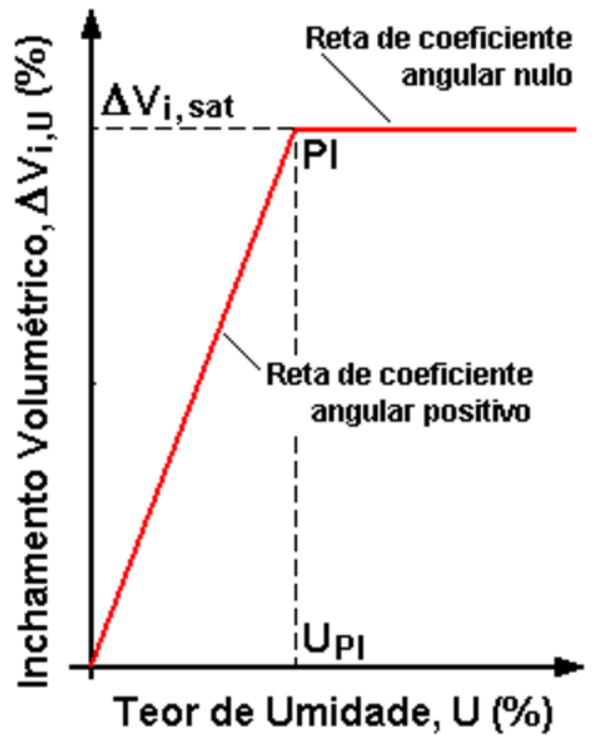

Fonte: LOGSDON e FINGER (2000)

$$
\begin{aligned}
& \text { Para } 0 \% \leq \mathrm{U}<\mathrm{U}_{\mathrm{PI}}: \Delta \mathrm{V}_{\mathrm{i}, \mathrm{U}}=\delta_{\mathrm{V} \mathrm{i}} \cdot \mathrm{U} \\
& \text { Para } \mathrm{U} \geq \mathrm{U}_{\mathrm{PI}}: \Delta \mathrm{V}_{\mathrm{i}, \mathrm{U}}=\Delta \mathrm{V}_{\mathrm{i}, \text { sat }}
\end{aligned}
$$

Nas quais: U é o teor de umidade da madeira, em um instante qualquer do ensaio; $\Delta \mathrm{V}_{\mathrm{i}, \mathrm{U}}$ e $\Delta \mathrm{V}_{\mathrm{i} \text {,sat }}$ são as variações volumétricas no inchamento, a partir de $\mathrm{U}=0 \%$, até madeira com um teor de umidade qualquer e na situação saturada em água $\left(\mathrm{U} \geq \mathrm{U}_{\mathrm{PI}}\right) ; \delta_{\mathrm{Vi}}$ é o coeficiente de inchamento volumétrico, que caracteriza o coeficiente angular da reta inicial do diagrama; $U_{P I}$ é o teor de umidade no ponto de interseção das duas retas do diagrama de inchamento.

Para o estudo da retração, praticamente todas as normas técnicas do passado admitiram o mesmo comportamento observado no inchamento. Isto foi um erro, pois o fenômeno não é diretamente associado ao teor de umidade, mas a uma parcela dele, ou seja, ao teor de água de impregnação, que por sua vez depende do histórico de umidade. 
Logsdon (2000) identificou as diferenças de comportamento entre inchamentos e retrações. Logsdon e Finger (2000) estabeleceram o modelo para o comportamento da madeira à retração volumétrica, apresentado na Figura 2 e nas Expressões (3) e (4), que também se verifica para as deformações específicas na secagem (retrações lineares).

$$
\begin{aligned}
& \text { Para } 0 \% \leq \mathrm{U}<\mathrm{U}_{\text {cond. }}: \\
& \Delta \mathrm{V}_{\mathrm{r}, \mathrm{U}}=\Delta \mathrm{V}_{\mathrm{r}, \text { cond. }} \cdot\left(\frac{\mathrm{U}}{\mathrm{U}_{\text {cond. }}}\right)^{\beta_{0, \mathrm{~V}}} \\
& \text { Para } \mathrm{U}_{\text {cond. }} \leq \mathrm{U} \leq \mathrm{U}_{\text {sat. }}: \\
& \text { Máximo }\left[\Delta \mathrm{V}_{\mathrm{r}, \mathrm{U}}=\Delta \mathrm{V}_{\mathrm{r}, \text { cond. }} \mathrm{e}\right. \\
& \left.\Delta \mathrm{V}_{\mathrm{r}, \mathrm{U}}=\Delta \mathrm{V}_{\mathrm{r}, \text { sat. }} \cdot\left(\frac{\mathrm{U}}{\mathrm{U}_{\text {sat. }}}\right)^{\beta_{1, \mathrm{~V}}}\right]
\end{aligned}
$$

Nas quais: U é o teor de umidade da madeira, em um instante qualquer do ensaio, em particular, $\mathrm{U}_{\text {sat. }}$ e $\mathrm{U}_{\text {cond. }}$ correspondem, respectivamente, aos teores de umidade do corpo-de-prova saturado em água e condicionado em clima padronizado (temperatura de $20^{\circ} \mathrm{C} \pm 2^{\circ} \mathrm{C}$ e umidade relativa do ar de $65 \% \pm 5 \%) ; \Delta \mathrm{V}_{\mathrm{r}, \mathrm{U}}$ é a variação volumétrica na retração, em um instante qualquer do ensaio, em particular, $\Delta \mathrm{V}_{\mathrm{r}, \text { sat. }} \quad$ e $\Delta \mathrm{V}_{\mathrm{r}, \mathrm{cond}} \quad$ correspondem, às variações volumétricas do corpo-de-prova saturado e condicionado, até seco $(\mathrm{U}=0 \%)$; $\beta_{0, \mathrm{~V}}$ e $\beta_{1, \mathrm{~V}}$ são os expoentes das curvas.

Figura 2 - Modelo de comportamento para retração volumétrica

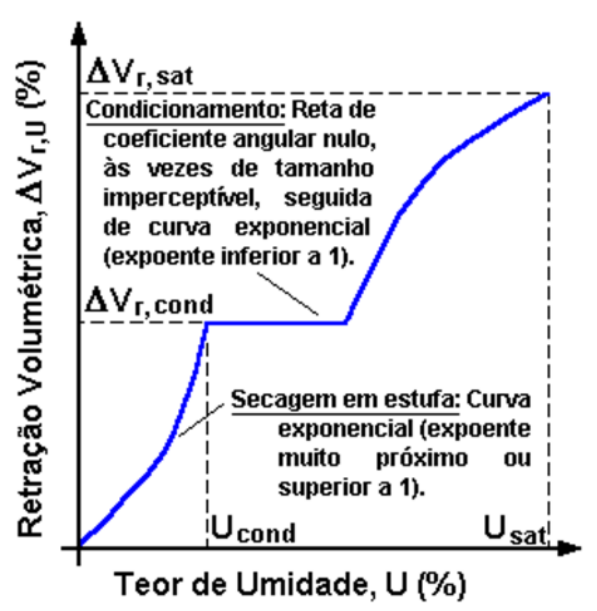

Fonte: LOGSDON e FINGER (2000)

Kollmann e Côté Jr. (1984) apresentam um estudo sobre a variação da densidade aparente $\left(\rho_{\mathrm{u}}\right)$ com o teor de umidade (U) durante o umedecimento da madeira, obtendo o modelo das Expressões (5) e (6), função da densidade aparente da madeira seca $\left(\rho_{0}\right)$ e de características do inchamento volumétrico.

$$
\begin{aligned}
& \text { Para } 0 \% \leq \mathrm{U}<\mathrm{U}_{\mathrm{PI}}: \\
& \rho_{\mathrm{u}}=\rho_{0} \cdot \frac{\left(1+\frac{\mathrm{U}}{100}\right)}{\left(1+\frac{\delta_{\mathrm{V}} \cdot \mathrm{U}}{100}\right)}
\end{aligned}
$$

$$
\begin{aligned}
& \text { Para } U \geq U_{P I}: \\
& \rho_{u}=\rho_{0} \cdot \frac{\left(1+\frac{U}{100}\right)}{\left(1+\frac{\Delta V_{i, \text { sat. }}}{100}\right)}
\end{aligned}
$$

Logsdon (2004), de forma análoga a Kollmann e Côté Jr. (1984), estudou a variação da densidade aparente com o teor de umidade durante a secagem da madeira, obtendo o modelo das Expressões (7) e (8), função de características da retração volumétrica.

$$
\begin{aligned}
& \text { Para } 0 \% \leq \mathrm{U}<\mathrm{U}_{\text {cond. }} \text { : } \\
& \rho_{\mathrm{u}}=\rho_{0} \cdot\left(1+\frac{\mathrm{U}}{100}\right) \cdot\left[1-\frac{\Delta \mathrm{V}_{\mathrm{r}, \text { cond. }}}{100} \cdot\left(\frac{\mathrm{U}}{\mathrm{U}_{\text {cond }}}\right)^{\beta_{0, \mathrm{~V}}}\right] \\
& \text { Para } \mathrm{U}_{\text {cond. }} \leq \mathrm{U} \leq \mathrm{U}_{\text {sat. }} \text { : }
\end{aligned}
$$




$$
\begin{aligned}
& \text { Mínimo }\left\{\rho_{\mathrm{u}}=\rho_{0} \cdot\left(1+\frac{\mathrm{U}}{100}\right) \cdot\left(1-\frac{\Delta \mathrm{V}_{\mathrm{r}, \text { cond }}}{100}\right) \mathrm{e}\right. \\
& \left.\rho_{\mathrm{u}}=\rho_{0} \cdot\left(1+\frac{\mathrm{U}}{100}\right) \cdot\left[1-\frac{\Delta \mathrm{V}_{\mathrm{r}, \text { sat. }}}{100} \cdot\left(\frac{\mathrm{U}}{\mathrm{U}_{\text {sat. }}}\right)^{\beta_{1, \mathrm{~V}}}\right]\right\}
\end{aligned}
$$

A densidade aparente é uma característica física fortemente relacionada às características mecânicas, portanto, obtida a densidade aparente da madeira é mecânica. Embora a classe de resistência seja definida pela resistência característica a compressão paralela às fibras, as classes de resistência, definidas no projeto de revisão da NBR 7190, da ABNT (2011), e apresentadas na Tabela 1, também apresentam valores esperados para a densidade aparente das classes. Assim, também é possível inferir sobre a classe de resistência a partir da densidade aparente.

Tabela 1 - Classes de resistência das madeiras

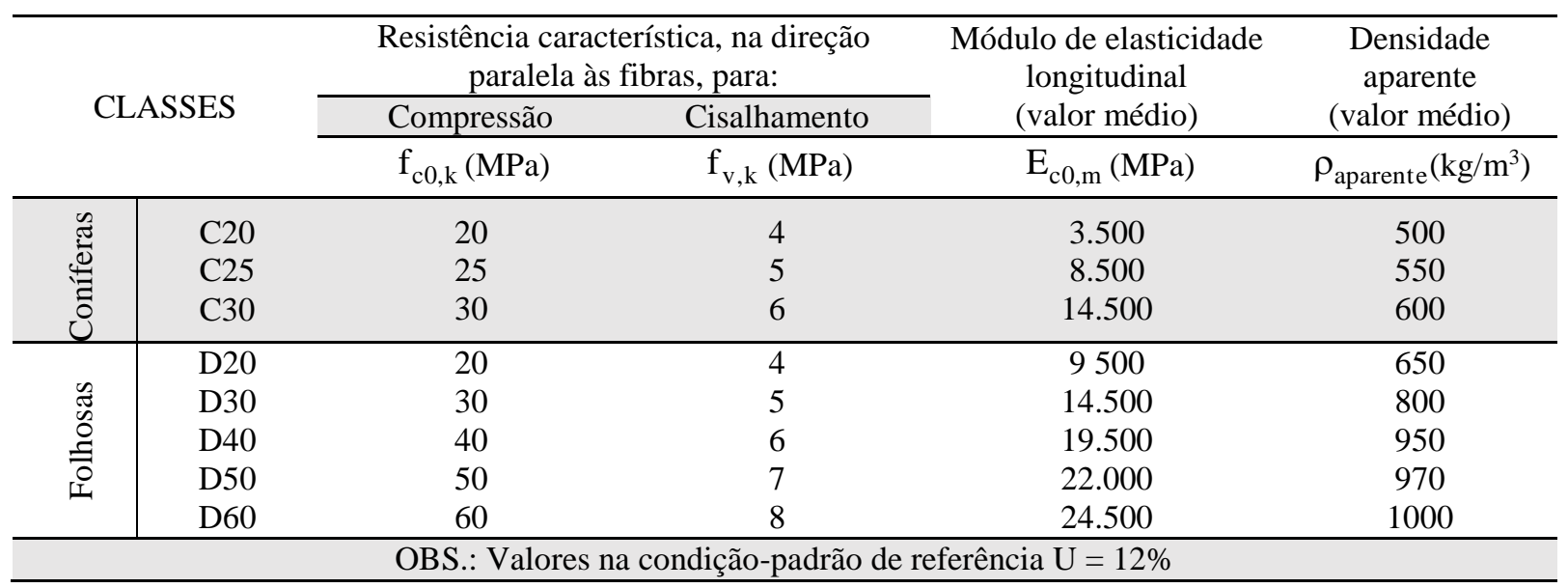

Fonte: Projeto de revisão da NBR 7190, da ABNT (2011)

O coeficiente de anisotropia, segundo Nock et al. (1975), é usado na indicação da qualidade da madeira quanto aos defeitos oriundos da secagem. Logsdon e Penna (2005) ampliaram os estudos de Nock et al. (1975) e apresentaram a Tabela 2. As Expressões (9) e (10) apresentam as definições dos coeficientes de anisotropia na retração e no inchamento.

$$
\begin{aligned}
& \mathrm{A}_{\mathrm{r}}=\frac{\varepsilon_{\mathrm{r}, 3, \text { sat }}}{\varepsilon_{\mathrm{r}, 2, \mathrm{sat}}} \\
& \mathrm{A}_{\mathrm{i}}=\frac{\varepsilon_{\mathrm{i}, 3, \text { sat }}}{\varepsilon_{\mathrm{i}, 2, \text { sat }}}
\end{aligned}
$$

Nas quais: $A_{r}$ é o coeficiente de anisotropia dimensional, na retração; $A_{i}$ é o coeficiente de anisotropia dimensional, no inchamento; $\varepsilon_{\mathrm{r}, 2 \text {,sat }}$ e $\varepsilon_{\mathrm{r}, 3, \text { sat }}$ são as deformações específicas do corpo-deprova, devido à retração, para uma variação no teor de umidade desde a situação saturado em água $\left(\mathrm{U}=\mathrm{U}_{\text {sat }}\right)$ até completamente seco $(\mathrm{U}=0 \%)$, respectivamente nas direções radial (2) e tangencial (3); $\quad \varepsilon_{\mathrm{i}, 2, \mathrm{sat}}$ e $\varepsilon_{\mathrm{i}, 3, \mathrm{sat}}$ são as deformações específicas do corpo-deprova, devido ao inchamento, para uma variação no teor de umidade desde a situação completamente seco $(U=0 \%)$ até saturado em água $\left(\mathrm{U}=\mathrm{U}_{\mathrm{sat}}\right)$, respectivamente nas direções radial (2) e tangencial (3). 
Tabela 2 - Coeficiente de anisotropia dimensional, qualidade e uso da madeira

\begin{tabular}{|c|c|c|c|}
\hline \multicolumn{2}{|c|}{ Coeficiente de anisotropia em: } & \multirow{2}{*}{$\begin{array}{l}\text { Qualidade da } \\
\text { madeira }\end{array}$} & \multirow{2}{*}{ Utilização indicada para a madeira } \\
\hline Retração, $A_{r}$ & Inchamento, $\mathrm{A}_{\mathrm{i}}$ & & \\
\hline Até 1,50 & Até 1,54 & Excelente & $\begin{array}{l}\text { Móveis finos, esquadrias, barcos, aparelhos musicais, } \\
\text { aparelhos de esporte e etc. }\end{array}$ \\
\hline 1,50 a 2,00 & 1,54 a 2,10 & Normal & $\begin{array}{l}\text { Estantes, mesas, armários, enfim usos que permitam } \\
\text { pequenos empenamentos. }\end{array}$ \\
\hline Acima de 2,00 & Acima de 2,10 & Ruim & $\begin{array}{l}\text { Construção civil (observadas as características } \\
\text { mecânicas), carvão, lenha etc. }\end{array}$ \\
\hline
\end{tabular}

Fonte: LOGSDON e PENNA (2005)

\section{MATERIAIS E MÉTODOS}

O material foi coletado de três arvores da espécie Cedro-marinheiro, Guarea trichilioides L., no município de Cotriguaçu - MT. De cada árvore foi coletado material botânico para a identificação e descrição da espécie, e um disco, na região do DAP (Diâmetro à Altura do Peito, ou seja, a 1,30 m da linha de afloramento da árvore junto ao solo), de aproximadamente $15 \mathrm{~cm}$ de espessura, do qual foi retirada uma pequena tábua diametral, de direções principais bem definidas, com $2 \mathrm{~cm}$ de espessura (na direção tangencial).

Para a caracterização dendrológica, foram preparadas exsicatas utilizando três exemplares da espécie além de informações sobre as particularidades do caule, da copa, das folhas, da casca e da madeira. A espécie foi identificada, pela comparação de exsicatas coletadas com material catalogado no Herbário Central da Universidade Federal de Mato Grosso e pela consulta a bibliografia especializada. A nomenclatura da espécie foi citada de acordo com o Index Kewensis.

Para a caracterização física, tomouse um lote formado por três árvores, de cada árvore foi retirado um disco na altura do DAP, dos quais foram extraídos 4 corpos-de-prova de dimensões $2 \mathrm{~cm} \mathrm{x}$ $3 \mathrm{~cm}$ x $5 \mathrm{~cm}$, respectivamente nas direções tangencial, radial e axial, resultando em 12 corpos-de-prova, posicionados aleatoriamente ao longo de uma linha diametral, correspondendo à amostragem mínima prevista na NBR 7190, da ABNT (1997), mantida no projeto de revisão da NBR 7190, da ABNT (2011). De cada árvore foi separado um corpo-de-prova como amostra de controle.

A caracterização física foi baseada na metodologia proposta por Logsdon (2002) para revisão da NBR 7190 da ABNT (1997), que inclui os ensaios de inchamento e retração. Também foram utilizados os conceitos e definições presentes na referida norma. Embora a redação seja diferente, o projeto de revisão da NBR 7190, da ABNT (2011), está em acordo com a metodologia utilizada.

O ensaio de estabilidade dimensional, proposto por Logsdon (2002), tem quatro fases:

1. Fase de secagem prévia, na qual os corpos-de-prova são condicionados aproximadamente $12 \%$ para início dos ensaios (U=U $\left.\mathrm{U}_{\text {início }} \cong 12 \%\right)$;

2. Fase de umedecimento, na qual os corpos-de-prova são saturados em água destilada $\left(\mathrm{U}=\mathrm{U}_{\mathrm{sat}}\right)$;

3. Fase de condicionamento, na qual os corpos-de-prova são novamente condicionados a aproximadamente $\quad 12 \%$ ( $\mathrm{U}=\mathrm{U}_{\text {cond }}$ );

4. Fase de secagem em estufa, onde os corpos-de-prova são completamente secos $(\mathrm{U}=0 \%)$. 
A fase de umedecimento e o instante do ensaio correspondente ao corpo-deprova completamente seco $(\mathrm{U}=0 \%)$ compõem o ensaio de inchamento. As fases de condicionamento e de secagem em estufa compõem o ensaio de retração. Logsdon (2002) fez uma descrição bastante detalhada do ensaio, que não será transcrita neste trabalho.

\section{RESULTADOS E DISCUSSÃO}

\section{Descrição Dendrológica}

A espécie Guarea trichilioides L., da família Meliaceae, é vulgarmente conhecida por Cedro-marinheiro e distribui-se naturalmente por toda a Região Amazônica, nas florestas ombrófilas aberta e densa, sobre solos argilosos, nos estados de Mato Grosso, Rondônia, Acre, Amazonas, Para e Roraima. Alcança mais de $50 \mathrm{~m}$ de altura e $80 \mathrm{~cm}$ de diâmetro. Nesta espécie o fuste é circular e muito cilíndrico, a ramificação é cimosa e a copa da árvore caracteristicamente capitataesférica. Suas folhas são alternas, compostas penadas-paripenadas, com gema terminal encurvada; folíolos aos pares opostos com base arredondada e ápice acuminado, glabros e lusídios. Sua casca é acastanhada, com manchas acinzentadas e geralmente recoberta por musgos; o ritidoma se desprende em placas e a casca viva é castanho-avermelhada, com odor marcante. A madeira dessa espécie, apresentada na Figura 3, é moderadamente pesada e compacta; de cor castanho-avermelhada; fácil de trabalhar recebendo bom acabamento e superfície lisa; de boa durabilidade natural; alburno e o cerne são distintos; apresenta textura média, grã regular; sem cheiro e o gosto é indistinto. Floresce nos meses de dezembro a março e frutifica de novembro a dezembro. Sua madeira é própria para carpintaria, marcenaria e construção em geral.

Figura 3 - Aspectos da madeira de Cedromarinheiro, Guarea trichilioides L.
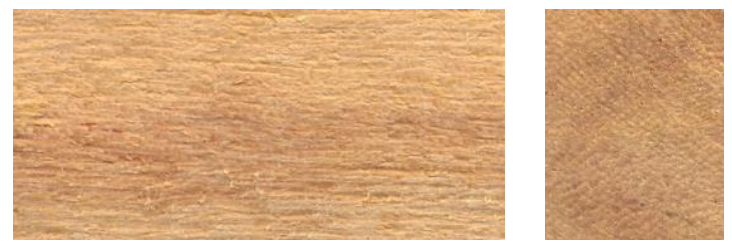

a) Vista longitudinal b) Vista transversal

\section{Principais Características Físicas}

As principais características físicas da madeira de Cedro-marinheiro, Guarea trichilioides L., obtidas neste trabalho, são apresentadas nas Tabelas 3 a 6 . A maioria dos valores, apresentados nas Tabelas 3 a 6, são necessários para a recuperação dos diagramas de inchamentos, retrações, e da variação da densidade aparente com o teor de umidade.

Por outro lado, o valor de $\rho_{\text {ap }, 12 \%} \cong 0,7506 \mathrm{~g} / \mathrm{cm}^{3}$ é indicativo da resistência mecânica e da qualidade da madeira para uso estrutural, já os valores de $\quad \mathrm{A}_{\mathrm{i}}=\varepsilon_{\mathrm{i}, 3} / \varepsilon_{\mathrm{i}, 2} \cong 2,0945$ e $\quad \mathrm{de}$ $\mathrm{A}_{\mathrm{r}}=\varepsilon_{\mathrm{r}, 3} / \varepsilon_{\mathrm{r}, 2} \cong 1,9965$, permitem estimar a qualidade da madeira quanto a defeitos de secagem e a alguns usos. 
Falavinha, A. F. et al., Estudo da estabilidade dimensional da madeira de Cedro-marinheiro, Guarea trichilioides L.. E\&S - Engineering and Science, (2016), 5:X

Tabela 3 - Características físicas da madeira de Cedro-marinheiro, Guarea trichilioides L., nos ensaios de inchamentos

\begin{tabular}{|c|c|c|c|c|c|c|c|}
\hline \multirow{3}{*}{ Corpo-de-prova } & \multirow{2}{*}{$\begin{array}{c}\text { U. de } \\
\text { saturação }\end{array}$} & \multicolumn{3}{|c|}{ Coeficiente de Inchamento: } & \multicolumn{3}{|c|}{ Inchamento total (madeira saturada) } \\
\hline & & Radial & Tangencial & Volumétrico & Radial & Tangencial & Volumétrico \\
\hline & $\mathrm{U}_{\text {sat }}(\%)$ & $\delta_{\mathrm{i}, 2}$ & $\delta_{\mathrm{i}, 3}$ & $\delta \mathrm{Vi}$ & $\varepsilon_{\mathrm{i}, 2}(\%)$ & $\varepsilon_{\mathrm{i}, 3}(\%)$ & $\Delta \mathbf{V}_{\mathrm{i}}(\%)$ \\
\hline CM-A1 & 61,63 & 0,1640 & 0,3225 & 0,5073 & 4,6372 & 10,2697 & 15,9606 \\
\hline CM-A2 & 59,21 & 0,1352 & 0,2723 & 0,4083 & 4,4542 & 8,7467 & 13,9968 \\
\hline CM-A3 & 68,30 & 0,1355 & 0,2622 & 0,3917 & 4,0829 & 8,9568 & 13,7068 \\
\hline CM-A4 & 61,59 & 0,1262 & 0,2979 & 0,4390 & 4,1250 & 9,2683 & 14,2405 \\
\hline CM-B1 & 57,94 & 0,1566 & 0,3455 & 0,5106 & 4,8311 & 9,7143 & 15,3158 \\
\hline CM-B2 & 58,27 & 0,1624 & 0,4574 & 0,6331 & 4,0328 & 11,6933 & 16,4813 \\
\hline CM-B3 & 56,65 & 0,1884 & 0,2505 & 0,4541 & 5,2248 & 10,2981 & 16,4145 \\
\hline CM-B4 & 60,09 & 0,1491 & 0,3497 & 0,5205 & 4,1022 & 10,2367 & 15,3320 \\
\hline $\mathrm{CM}-\mathrm{C} 1$ & 64,25 & 0,1943 & 0,3148 & 0,5210 & 5,1485 & 9,0264 & 14,9208 \\
\hline $\mathrm{CM}-\mathrm{C} 2$ & 56,83 & 0,1941 & 0,2758 & 0,4864 & 5,5011 & 8,4619 & 14,8991 \\
\hline $\mathrm{CM}-\mathrm{C} 3$ & 58,39 & 0,1391 & 0,2828 & 0,4202 & 4,7544 & 8,8776 & 14,1178 \\
\hline CM-C4 & 55,35 & 0,1604 & 0,2460 & 0,4276 & 4,9922 & 9,8818 & 17,9858 \\
\hline Número & 12 & 12 & 12 & 12 & 12 & 12 & 12 \\
\hline Média & 59,87 & 0,1588 & 0,3065 & 0,4767 & 4,6572 & 9,6193 & 15,2810 \\
\hline Desvio Padrão & 3,6358 & 0,0235 & 0,0588 & 0,0674 & 0,5027 & 0,9150 & 1,2557 \\
\hline \multicolumn{8}{|c|}{ INTERVALO DE CONFIANÇA DA MÉDIA } \\
\hline$t(95 \%)$ & 2,2010 & 2,2010 & 2,2010 & 2,2010 & 2,2010 & 2,2010 & 2,2010 \\
\hline Lim. Inferior & $\mathbf{5 7 , 5 6}$ & $\mathbf{0 , 1 4 3 9}$ & 0,2691 & $\mathbf{0 , 4 3 3 8}$ & 4,3378 & 9,0379 & 14,4831 \\
\hline Lim. Superior & 62,18 & $\mathbf{0 , 1 7 3 7}$ & $\mathbf{0 , 3 4 3 8}$ & 0,5195 & 4,9766 & 10,2007 & 16,0789 \\
\hline
\end{tabular}

Tabela 4 - Características físicas de Cedro-marinheiro, Guarea trichilioides L., na fase de condicionamento dos ensaios de retrações

\begin{tabular}{|c|c|c|c|c|c|c|}
\hline \multirow{3}{*}{ Corpo-de-prova } & \multicolumn{3}{|c|}{ Retração total (madeira saturada) } & \multicolumn{3}{|c|}{ Expoentes no trecho final (retrações) } \\
\hline & Radial & Tangencial & Volumétrica & Radial & Tangencial & Volumétrica \\
\hline & $\varepsilon \mathrm{r}, 2$, sat $(\%)$ & $\varepsilon_{\mathrm{r}, 3, \text { sat }}(\%)$ & $\Delta \mathbf{V}_{\mathrm{r}, \text { sat }}(\%)$ & $\beta_{1,2}$ & $\beta_{1,3}$ & $\beta_{1, \mathrm{~V}}$ \\
\hline CM-A1 & 4,4317 & 9,3133 & 13,7638 & 0,0975 & 0,1704 & 0,1506 \\
\hline CM-A2 & 4,2643 & 8,0432 & 12,2782 & 0,1676 & 0,1946 & 0,1952 \\
\hline CM-A3 & 3,9228 & 8,2205 & 12,0545 & 0,1630 & 0,1756 & 0,1608 \\
\hline CM-A4 & 3,9616 & 8,4821 & 12,4653 & 0,1706 & 0,2139 & 0,1930 \\
\hline CM-B1 & 4,6084 & 8,8542 & 13,2816 & 0,1648 & 0,1677 & 0,1706 \\
\hline CM-B2 & 3,8764 & 10,4691 & 14,1493 & 0,0821 & 0,1141 & 0,1103 \\
\hline CM-B3 & 4,9654 & 9,3366 & 14,1000 & 0,1439 & 0,2385 & 0,2039 \\
\hline CM-B4 & 3,9406 & 9,2861 & 13,2938 & 0,1890 & 0,1886 & 0,1782 \\
\hline $\mathrm{CM}-\mathrm{C} 1$ & 4,8964 & 8,2791 & 12,9836 & 0,1513 & 0,1787 & 0,1604 \\
\hline $\mathrm{CM}-\mathrm{C} 2$ & 5,2143 & 7,8018 & 12,9671 & 0,1339 & 0,1819 & 0,1649 \\
\hline $\mathrm{CM}-\mathrm{C} 3$ & 4,5386 & 8,1537 & 12,3712 & 0,0972 & 0,1310 & 0,1323 \\
\hline $\mathrm{CM}-\mathrm{C} 4$ & 4,7548 & 8,9932 & 15,2440 & 0,1308 & 0,2162 & 0,3524 \\
\hline Número & 12 & 12 & 12 & 12 & 12 & 12 \\
\hline Média & 4,4479 & 8,7694 & 13,2461 & 0,1410 & 0,1809 & 0,1811 \\
\hline Desvio Padrão & 0,4587 & 0,7567 & 0,9384 & 0,0337 & 0,0346 & 0,0601 \\
\hline \multicolumn{7}{|c|}{ INTERVALO DE CONFIANÇA DA MÉDIA } \\
\hline $\mathbf{t}(\mathbf{9 5 \%})$ & 2,2010 & 2,2010 & 2,2010 & 2,2010 & 2,2010 & 2,2010 \\
\hline Lim. Inferior & 4,1565 & 8,2886 & 12,6498 & 0,1196 & 0,1590 & 0,1429 \\
\hline Lim. Superior & 4,7394 & 9,2502 & 13,8423 & 0,1624 & 0,2029 & 0,2193 \\
\hline
\end{tabular}


Falavinha, A. F. et al., Estudo da estabilidade dimensional da madeira de Cedro-marinheiro, Guarea trichilioides L.. E\&S - Engineering and Science, (2016), 5:X.

Tabela 5 - Características físicas de Cedro-marinheiro, Guarea trichilioides L., na fase de secagem dos ensaios de retrações

\begin{tabular}{|c|c|c|c|c|c|c|c|}
\hline \multirow[t]{3}{*}{ Corpo-de-prova } & \multirow{2}{*}{$\begin{array}{l}\text { Umidade de } \\
\text { condicio- } \\
\text { namento }\end{array}$} & \multicolumn{3}{|c|}{$\begin{array}{c}\text { Retrações para madeira } \\
\text { Condicionada } \\
\end{array}$} & \multicolumn{3}{|c|}{$\begin{array}{c}\text { Expoentes no trecho } \\
\text { inicial (retrações) }\end{array}$} \\
\hline & & Radial & Tangencial & Volumétrica & Radial & Tang. & Vol. \\
\hline & $\mathrm{U}_{\text {cond }}(\%)$ & $\varepsilon_{\mathrm{r}, 2, \text { cond }}(\%)$ & $\varepsilon_{\mathrm{r}, 3, \text { cond }}(\%)$ & $\Delta \mathrm{V}_{\mathrm{r}, \text { cond }}(\%)$ & $\beta_{0,2}$ & $\beta_{0,3}$ & $\beta_{0, \mathrm{~V}}$ \\
\hline CM-A1 & 14,70 & 2,4615 & 4,9310 & 7,6307 & 1,4215 & 2,0020 & 1,6925 \\
\hline CM-A2 & 13,64 & 2,4778 & 4,2390 & 6,7873 & 1,6842 & 2,7443 & 2,2343 \\
\hline CM-A3 & 14,74 & 2,2113 & 3,8501 & 6,1722 & 1,5382 & 1,6063 & 1,5921 \\
\hline CM-A4 & 14,83 & 2,2901 & 3,1496 & 5,6429 & 1,4277 & 1,2602 & 1,2797 \\
\hline CM-B1 & 14,71 & 2,6736 & 4,8913 & 7,5897 & 1,3779 & 1,5791 & 1,4441 \\
\hline CM-B2 & 14,56 & 2,3685 & 4,0907 & 7,4069 & 1,2916 & 1,9395 & 1,7000 \\
\hline CM-B3 & 14,24 & 2,7410 & 5,0437 & 7,8570 & 1,6864 & 1,2316 & 1,3704 \\
\hline CM-B4 & 14,50 & 2,1309 & 4,7407 & 7,0819 & 1,4401 & 2,7358 & 2,1551 \\
\hline CM-C1 & 14,82 & 2,9132 & 4,5499 & 7,5399 & 1,4682 & 1,6130 & 1,5513 \\
\hline $\mathrm{CM}-\mathrm{C} 2$ & 14,96 & 3,0349 & 4,4157 & 6,7569 & 1,5215 & 2,7338 & 1,8183 \\
\hline CM-C3 & 15,28 & 2,7435 & 4,6229 & 7,1876 & 1,6599 & 2,2144 & 1,9954 \\
\hline $\mathrm{CM}-\mathrm{C} 4$ & 14,65 & 2,7778 & 4,4638 & 7,3297 & 1,7415 & 1,6137 & 1,5669 \\
\hline Número & 12 & 12 & 12 & 12 & 12 & 12 & 12 \\
\hline Média & 14,64 & 2,5687 & 4,4157 & 7,0819 & 1,5215 & 1,9395 & 1,7000 \\
\hline Desvio Padrão & 0,4020 & 0,2873 & 0,5321 & 0,6494 & 0,1425 & 0,5566 & 0,3009 \\
\hline \multicolumn{8}{|c|}{ INTERVALO DE CONFIANÇA DA MÉDIA } \\
\hline $\mathbf{t}(\mathbf{9 5 \%})$ & 2,2010 & 2,2010 & 2,2010 & 2,2010 & 2,2010 & 2,2010 & 2,2010 \\
\hline Lim. Inferior & 14,38 & 2,3862 & 4,0776 & 6,6693 & 1,4310 & $\mathbf{1 , 5 8 5 8}$ & $\mathbf{1 , 5 0 8 8}$ \\
\hline Lim. Superior & 14,89 & 2,7512 & 4,7538 & 7,4945 & 1,6121 & 2,2932 & $\mathbf{1 , 8 9 1 2}$ \\
\hline
\end{tabular}

Tabela 6 - Outras características físicas da madeira de Cedro-marinheiro, Guarea trichilioides L.

\begin{tabular}{|c|c|c|c|c|c|c|}
\hline \multirow[t]{3}{*}{ Corpo-de-prova } & \multirow{2}{*}{$\begin{array}{c}\text { Umidade } \\
\text { Ponto de } \\
\text { Interseção }\end{array}$} & \multicolumn{2}{|c|}{$\begin{array}{c}\text { Densidade aparente } \\
\text { da madeira } \\
\end{array}$} & \multirow{2}{*}{$\begin{array}{l}\text { Densidade } \\
\text { básica }\end{array}$} & \multicolumn{2}{|c|}{$\begin{array}{c}\text { Coeficiente de } \\
\text { Anisotropia em } \\
\end{array}$} \\
\hline & & Seca $(0 \%)$ & A $12 \%$ & & Inchamento & Retração \\
\hline & $\mathrm{U}_{\mathrm{PI}}(\%)$ & $\rho_{a p, 0 \%}\left(\mathrm{~g} / \mathrm{cm}^{3}\right)$ & $\rho_{a p, 12 \%}\left(\mathrm{~g} / \mathrm{cm}^{3}\right)$ & Pbas $\left(\mathrm{g} / \mathrm{cm}^{3}\right)$ & $\mathbf{A}_{\mathbf{i}}$ & $\mathbf{A}_{\mathbf{r}}$ \\
\hline CM-A1 & 31,46 & 0,7303 & 0,7737 & 0,6298 & 2,2146 & 2,1015 \\
\hline CM-A2 & 34,28 & 0,6718 & 0,7140 & 0,5893 & 1,9637 & 1,8862 \\
\hline CM-A3 & 34,99 & 0,6393 & 0,6842 & 0,5623 & 2,1937 & 2,0956 \\
\hline CM-A4 & 32,43 & 0,6709 & 0,7191 & 0,5873 & 2,2469 & 2,1411 \\
\hline CM-B1 & 30,00 & 0,7280 & 0,7692 & 0,6313 & 2,0108 & 1,9213 \\
\hline CM-B2 & 31,09 & 0,7242 & 0,7678 & 0,6217 & 2,8996 & 2,7007 \\
\hline CM-B3 & 36,14 & 0,7342 & 0,7712 & 0,6306 & 1,9710 & 1,8803 \\
\hline CM-B4 & 29,45 & 0,7244 & 0,7731 & 0,6281 & 2,4954 & 2,3565 \\
\hline $\mathrm{CM}-\mathrm{C} 1$ & 28,64 & 0,6917 & 0,7326 & 0,6019 & 1,7532 & 1,6909 \\
\hline $\mathrm{CM}-\mathrm{C} 2$ & 30,63 & 0,7211 & 0,7711 & 0,6276 & 1,5382 & 1,4962 \\
\hline CM-C3 & 33,60 & 0,7066 & 0,7563 & 0,6192 & 1,8672 & 1,7965 \\
\hline $\mathrm{CM}-\mathrm{C} 4$ & 33,23 & 0,7310 & 0,7748 & 0,6196 & 1,9795 & 1,8914 \\
\hline Número & 12 & 12 & 12 & 12 & 12 & 12 \\
\hline Média & 32,16 & 0,7061 & 0,7506 & 0,6124 & 2,0945 & 1,9965 \\
\hline Desvio Padrão & 2,3357 & 0,0308 & 0,0305 & 0,0222 & 0,3550 & 0,3153 \\
\hline \multicolumn{7}{|c|}{ INTERVALO DE CONFIANÇA DA MÉDIA } \\
\hline $\mathbf{t}(\mathbf{9 5 \%})$ & 2,2010 & 2,2010 & 2,2010 & 2,2010 & 2,2010 & 2,2010 \\
\hline Lim. Inferior & 30,68 & 0,6866 & 0,7312 & $\mathbf{0 , 5 9 8 3}$ & 1,8689 & 1,7962 \\
\hline Lim. Superior & 33,65 & $\mathbf{0 , 7 2 5 7}$ & $\mathbf{0 , 7 7 0 0}$ & 0,6265 & 2,3201 & 2,1968 \\
\hline
\end{tabular}


O modelo proposto por Kollmann e Côté Jr. (1984), para o diagrama de inchamentos, apresentou regressão $\left(\mathrm{F}_{\text {calculado }}>\mathrm{F}_{\text {de }}\right.$ significação $)$. $\mathrm{O}$ ajuste, dos resultados ao modelo, pode ser considerado muito bom, pois os coeficientes de determinação obtidos situam-se no intervalo $0,8229 \leq \mathrm{R}^{2} \leq 0,9853$.

O modelo proposto por Logsdon e Finger (2000), para o diagrama de retrações, apresentou regressão $\left(\mathrm{F}_{\text {calculado }}>\right.$ $\mathrm{F}_{\mathrm{de}}$ significação). $\mathrm{O}$ ajuste, dos resultados ao modelo, pode ser considerado muito bom, pois os coeficientes de determinação obtidos situam-se no intervalo $0,8773 \leq \mathrm{R}^{2} \leq 0,9850$.

O modelo matemático proposto por Logsdon (2004), para a variação da densidade aparente com o teor de umidade no umedecimento, função do modelo proposto por Kollmann e Côté Jr. (1984) para o diagrama de inchamentos, apresentou excelente ajuste aos dados experimentais.

O modelo matemático proposto por Logsdon (2004), para a variação da densidade aparente com o teor de umidade na secagem, função do modelo proposto por Logsdon e Finger (2000) para o diagrama de retrações, apresentou ajuste muito bom aos dados experimentais.

Utilizando-se as médias das principais características físicas, apresentadas nas Tabelas 3 a 6, podem-se construir as curvas apresentadas nas Figuras 4 a 7 , que podem ser interpretadas, e utilizadas, como correspondentes ao comportamento médio da espécie.

A comparação das curvas médias, representativas da variação da densidade aparente com o teor de umidade, no umedecimento e na secagem, apresentadas na Figura 8, permite observar comportamentos distintos, da madeira dessa espécie, conforme o sentido da variação da umidade.

Figura 4 - Comportamento médio do diagrama de inchamentos da madeira de Cedro-marinheiro, Guarea trichilioides L.

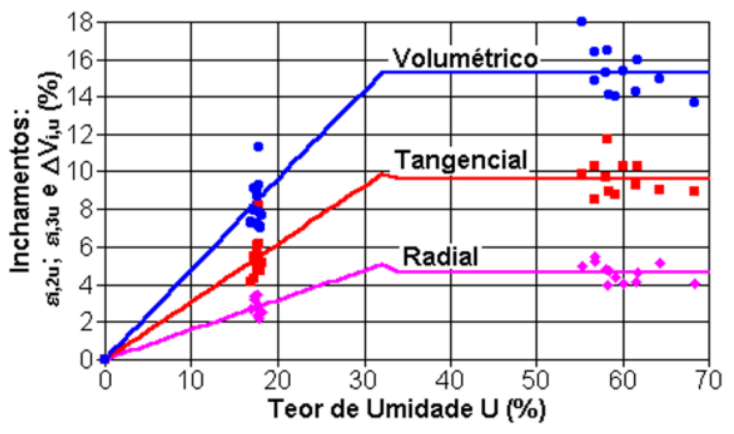

Figura 5 - Comportamento médio do diagrama de retrações da madeira de Cedro-marinheiro, Guarea trichilioides $\mathrm{L}$.

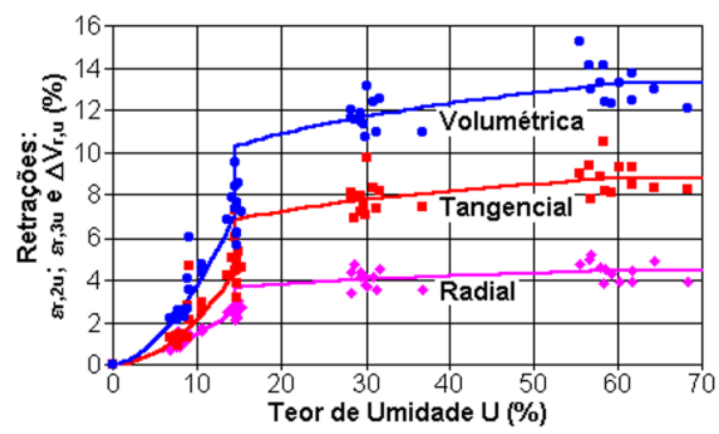

Figura 6 - Comportamento médio da variação da densidade aparente, com o teor de umidade, em um processo de umedecimento da madeira de Cedromarinheiro, Guarea trichilioides L.

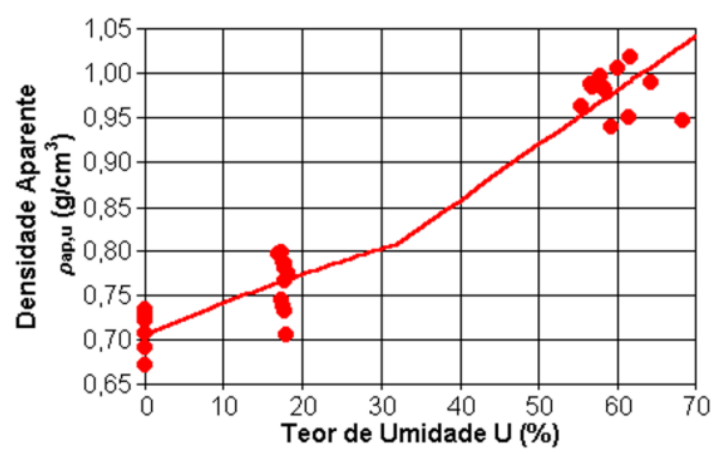

Na década de 1960, durante o Projeto RADAM, a espécie avaliada neste trabalho e outras espécies da Amazônia, foram caracterizadas pelo Instituto de Pesquisas Tecnológicas, IPT, de São Paulo (IPT, 2013). Entretanto o método utilizado 
continha erros no ensaio de retração, pois partiam de hipótese errada. Assim, consideram-se originais os resultados deste trabalho e a comparação com os dados obtidos anteriormente (errados) não faz sentido.

Figura 7 - Comportamento médio da variação da densidade aparente, com o teor de umidade, em um processo de secagem da madeira de Cedromarinheiro, Guarea trichilioides L.

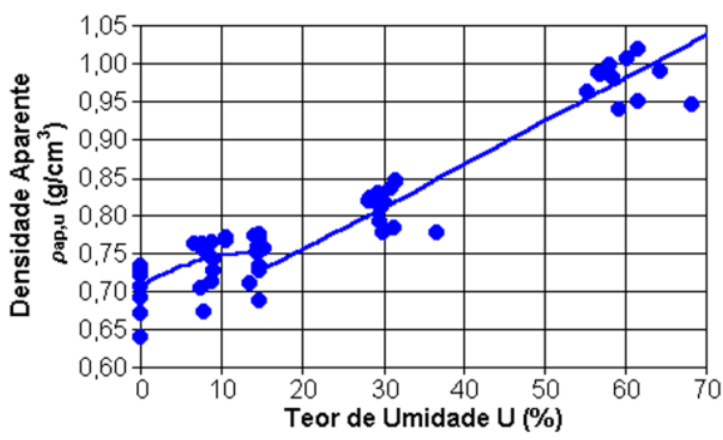

Figura 8 - Estudo comparativo da variação da densidade aparente, com o teor de umidade, ao longo dos processos de umedecimento e secagem, para a madeira de Cedro-marinheiro, Guarea trichilioides $\mathrm{L}$.

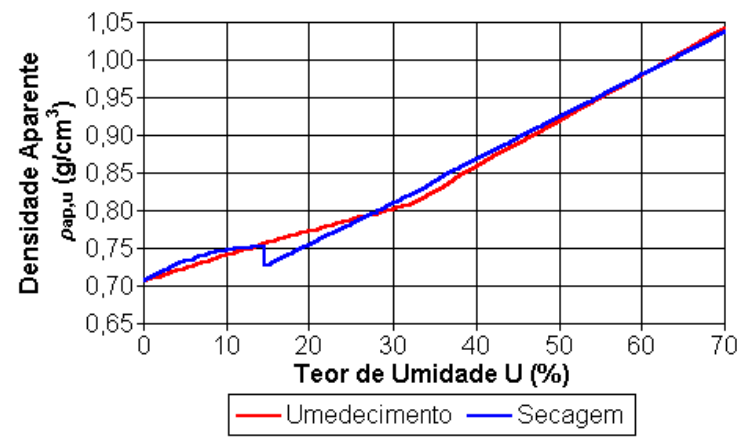

\section{CONCLUSÕES}

Os valores médios de densidade aparente $\left(\rho_{\mathrm{ap}, 12 \%}=0,7506 \mathrm{~g} / \mathrm{cm}^{3}\right)$ e de densidade básica $\left(\rho_{\mathrm{ba}}=0,6124 \mathrm{~g} / \mathrm{cm}^{3}\right)$ indicam que a madeira de Cedromarinheiro, Guarea trichilioides L., possui mediana densidade e sugerem a classe de resistência D30 ou D40 das dicotiledôneas, definida no projeto de revisão da NBR 7190 (ABNT, 2011), permitindo recomendar a madeira para aplicação em estruturas de madeira de médio porte.
Os coeficientes de anisotropia dimensional no inchamento, $\mathrm{A}_{\mathrm{i}}=2,0945$, e na retração, $A_{r}=1,9965$, indicam que a madeira de Cedro-marinheiro, Guarea trichilioides L., é considerada normal, podendo ser utilizada na fabricação de móveis que aceitem pequenos empenamentos, como mesas, cadeiras, estantes etc.

As Tabelas 3 a 6 apresentadas anteriormente, fornecem dados que podem ser utilizados, como indicativos de qualidade, por outros setores da indústria da madeira.

\section{AGRADECIMENTOS}

À Fundação de Amparo à Pesquisa do Estado de Mato Grosso (FAPEMAT), pelo financiamento do projeto que deu origem a este trabalho.

\section{REFERÊNCIAS BIBLIOGRÁFICAS}

ASSOCIAÇÃO BRASILEIRA DE NORMAS TÉCNICAS. NBR 7190 Projeto de Estruturas de Madeiras. Rio de Janeiro, 1997, 107p.

ASSOCIAÇÃO BRASILEIRA DE NORMAS TÉCNICAS. Projeto de revisão da NBR 7190 - Projeto de Estruturas de Madeiras. Rio de Janeiro, 2011.

INSTITUTO DE PESQUISAS TECNOLÓGICAS DO ESTADO DE SÃO PAULO (IPT). Catálogo de madeiras brasileiras para a construção civil. São Paulo. 2013.

KOLlMANN, F. F. P.; CÔTÉ JR., W. A. Principles of wood science and technology. Vol. I. Solid Wood. Reprint Springer-Verlag: Berlin, Heidelberg, New York, Tokyo. 1984, 592p. 
Falavinha, A. F. et al., Estudo da estabilidade dimensional da madeira de Cedro-marinheiro, Guarea trichilioides L.. E\&S - Engineering and Science, (2016), 5:X

LOGSDON, N. B. Estabilidade dimensional: Propostas para revisão da NBR 7190/97. In.: Encontro Brasileiro em Madeiras e em Estruturas de Madeira, , 8., 2002, Uberlândia. Anais... Uberlândia: UFU, 2002. 1 CD-ROM.

LOGSDON, N. B. Sobre os ensaios de retrações e inchamentos. Madeira: Arquitetura e Engenharia, ano 1, n. 2, p. 19-24, 2000.

LOGSDON, N. B. Variação da densidade aparente da madeira com sua umidade, modelagem teórico experimental. Madeira: Arquitetura e Engenharia, ano 4, n. 12, CD-ROM, 2004.

LOGSDON, N. B.; FINGER, Z. Modelos para especificação das curvas dos diagramas de retrações e inchamentos. Madeira: Arquitetura e Engenharia, ano 1, n. 3, CD-ROM, 2000.

LOGSDON, N. B.; PENNA, J. E. Comparison among the coefficients of dimensional anisotropy in swelling and in shrinkage. In: International Symposium on Wood Science and Technologies. IAWPS2005. Pacifico Yokohama, Yokohama. Annals... Pacifico Yokohama, Yokohama, Japan. 2005. p. 57-58.

NOCK, H. P.; RICHTER, H. G.; BURGER, L. M. Tecnologia da Madeira. Departamento de Engenharia e Tecnologia Rurais, Universidade Federal do Paraná, Curitiba, PR. 1975. 216p. 